\title{
AUTOMERLIN MOBILE ROBOT'S BILATERAL TELECONTROL WITH RANDOM DELAY
}

\author{
Aamir Shahzad \\ Department of Mechanical Engineering, The University of Lahore, Lahore, \\ (Pakistan) \\ E-mail: aamir.shahzad1@me.uol.edu.pk \\ Muhammad Salahudin \\ Department of Mechanical Engineering, The University of Lahore, Lahore, \\ (Pakistan) \\ E-mail: muhammadsalahuddin@hotmail.com \\ Iqbal Hussain \\ Department of Mechanical Engineering, The University of Lahore, Lahore, \\ (Pakistan) \\ E-mail:iqbal.hussain@me.uol.edu.pk
}




\section{ABSTRACT}

The main focus of this work is to design a bilateral telecontrol of a mobile robot AutoMerlin through the Internet. The Internet has an inherent delay, packet drop, out of order data transmission, duplication, and other impediments as a communication channel. These factors cause the system to become unstable and difficult to control through the Internet. The velocity tracking becomes really hard and the force feedback also rises to an unacceptable level due to delay and other impediments. In order to address these issues, a power based TDPC (Time Domain Passivity Control) has been utilized in this work for the development of stable telecontrol. This approach is based on energy. The energy has been classified as positive and negative energy to make passivity analysis independent of monitoring of net system energy in real time. Thus, monitoring the net energy output at each port enables the extension of TDPC for delayed systems called TDPN (Time Delay Power Network). TDPN helps in velocity/force tracking. It transmits velocity/force unaltered by rejecting the active energy. PO (Passivity Observers) indicate the active behavior and the PC (Passivity Controllers) dissipate extra surplus energy to keep the system stable and passive all times. The performance has been tested and plotted to show the effectiveness of the bilateral controller under random delay and other limitations.

\section{KEYWORDS}

Telecontrol; Haptic force; Joystick; Unstructured environment; Slave robot; Time delay power network; Random delay.

\section{INTRODUCTION}

Bilateral telecontrol of a mobile robot can be defined as control of the robot from a remote location while receiving force feedback from it as shown in Figure 1. The complete configuration of telecontrol comprises of a human operator, a haptic device connected to a computer having client algorithm, communication medium, slave robot equipped with server algorithm and remote environment [1-4]. The human operator applies the required maneuvers to the master haptic device which translates it into inputs for the slave robot in the remote environment. The desired inputs of the master device travel through some communication medium to the slave robot. These commands/instructions are executed by the slave robot in order to manipulate the remote environment. The effect of the environment is a reactive force on the slave robot. This force is sent as force feedback from a remote location to master haptic device. This force feedback is played over the haptic joystick. The human operator experiences the force feedback via haptic device and gets a sensation of actually manipulating the remote location directly. Telecontrol is a combination of different subsystems that exchange energy. The energy is exchanged by forwarding velocity and receiving force feedback [5-7]. Passivity control is based on system's net energy and it is an efficient tool for the stability analysis of the bilateral telecontrol. The combination of passive subsystems is always a passive system [8]. Hence, to ensure the passivity of the system, the subsystems can be analyzed to be passive all times. The stability based on the mathematical model of the system imposes conservative and strict rules on the 
performance of the system. It cannot easily tolerate the delay in the system and also requires accurate information about the remote environment [9-10]. The passivity is independent of a mathematical model of the system and is based on energy balance.

The master and slave are passive as they dissipate energy but when they are connected by means of any communication medium, then that medium can behave actively by inducing surplus energy in the system. The time delay is the main source of instability and activity too. A passivity-based approach using wave variables has been proposed by Niemeyer [11]. Wave variables have been utilized to develop the teleoperation with force reflection. Similarly, the scattering approach has been presented by Anderson and Spong [12]. These approaches have guaranteed the passive telecontrol at the cost of over dissipation of energy. This over dissipation of energy resulted in conservative performance [13-15]. To cope with these issues a remarkable approach based on TDPC has been presented by Ryu and Hannaford [16]. TDPC has two main elements called PO (Passivity Observer) and PC (Passivity Controller). PO keeps the track of energy entering and leaving the system to estimate net energy. The PC takes all the needed measures to dissipate surplus energy introduced in the control loop by various means. TDPC is based on calculation of net energy in real time to perform necessary control action. But in case of time delay due to distance or communication through a shared medium, the observation of net energy is not possible in real time. Hence the controller cannot take any action against the active energy. Therefore, Artigas has presented an extension to TDPC for the delayed system [17]. By using an Electrical/ Mechanical analogy, it has been proven that instability occurs due to non-passive communication block during
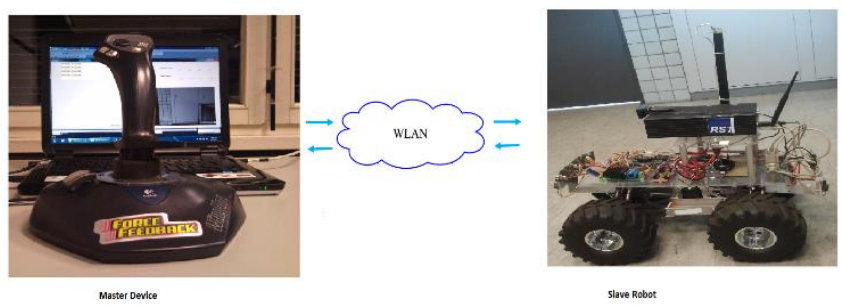

Figure 1. The bilateral telecontrol of AutoMerlin.

bilateral telecontrol with constant or random delay. A communication block has been modelled as a lossless entity which acts as a passive element even with time delay [18]. Teleoperator's stability has been guaranteed without limiting the bandwidth. This control approach ensures passivity of all subsystems in the closed-loop i.e. master, communication block and slave. Once the passivity is attained, the system is stable even though there are limitations and disturbances. These limitations are human operator dynamics, variable and unknown communication delay. Force feedback has been modelled as summation of two forces i.e. the virtual force and friction. The virtual force is based obstacles in the vicinity of the robot in a remote environment and friction is between ground and wheels. The virtual force calculated due to obstacles is a function of distance and velocity travelled by mobile robot. The complete method and its elaboration have been given in [4]. This force acts on the robot 
while it is moving in a remote environment is played over the haptic device as force feedback to the human operator. The velocity, force telecontrol is shown in Fig. 2. The slave robot is receiving the velocity command from the master device and delayed environmental force from the slave is reflected back.

Section I of this paper is Introduction about telecontrol, different approaches used in teleoperation and their pros and cons. Problem description has been presented in Section II with different plots to clearly illustrate the issues. Section III briefly describes the TDPN. It has mathematical modelling for the passivity of the network with random delay. The energy relations have been explained in it. Section IV has experimental results to show the performance of the teleoperation with TDPN. Section V has Conclusion and Future Work.

\section{PROBLEM DESCRIPTION}

The objective of telecontrol is to establish a close coordination among the interacting subsystems i.e. the haptic device and slave robot along with some limitations like limited bandwidth and random or constant time delay. Therefore, to elaborate the problem, there are some plots which have been included in this section to realize the actual issues. The blue line in the Figure 3 , shows the master haptic device's velocity and the green line represents the velocity of the slave robot. It is vivid that the slave velocity is more than the desired velocity set by the master device. This is due to the activity of the Internet because the master velocity travels through it and it has the delay in it and also other limitations like duplication, drop in packets and change in the order of data etc. [4]. The blue line indicates energy input at the master side while the green line is showing energy output and net energy is represented by a red line in Figure 4.

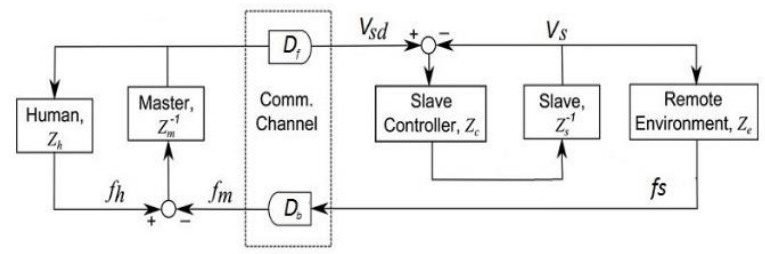

Figure 2. Block diagram of telecontrol.

It is clear from the plot that the net energy is negative and it is accumulating because active energy is not being dissipated. Figure 5, shows the two forces i.e. virtual force on the slave robot and force feedback on master haptic device. During certain intervals like after 4 seconds, the force feedback is larger as compared to the environmental force acting on the slave. Due to surge in force, the energy output is greater as compared to the energy input. This implies that the shared medium i.e. Internet is adding energy into the system to make it active and unstable. The energy comparison between net energy, input energy and output energy from slave to master has been plotted in the Figure 6. 


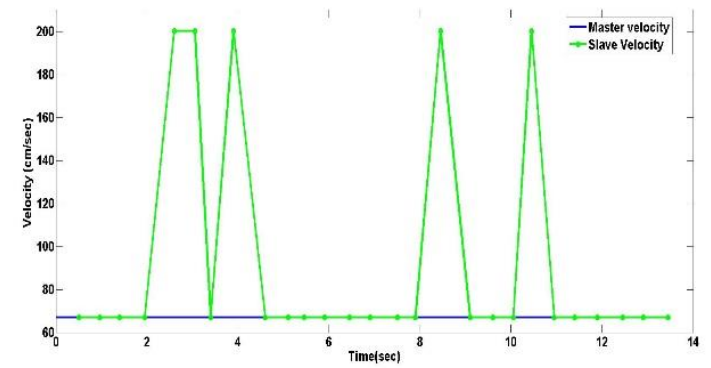

Figure 3. Linear velocities of both robots.

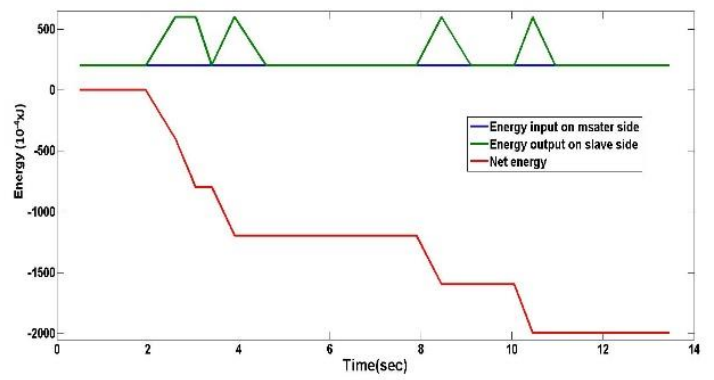

Figure 4. Energy flow master to slave.

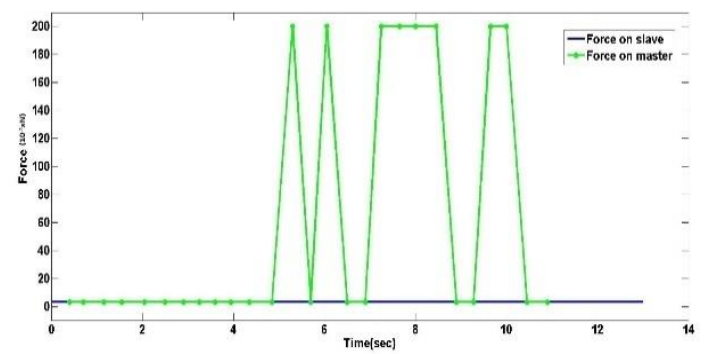

Figure 5. Force comparison on both robots.

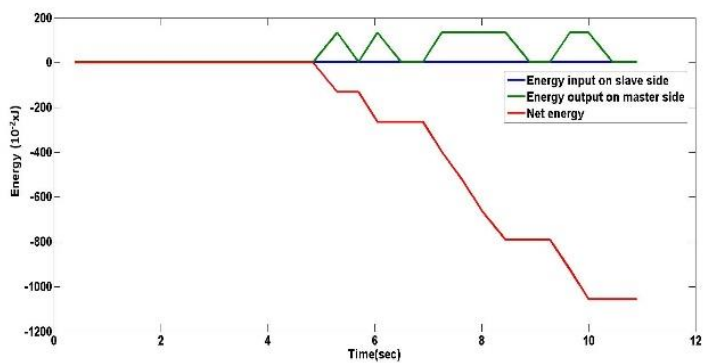

Figure 6. Energy flow slave to master.

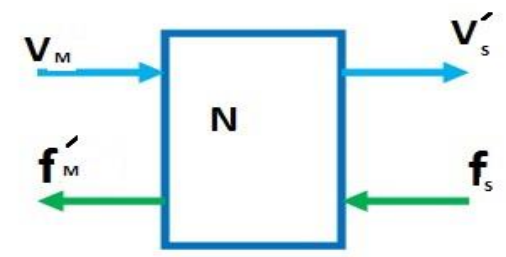

Figure 7. A Two-port network as TDPN.

\section{TDPN (TIME DELAY POWER NETWORK)}

The communication medium is adding active energy in the passive telecontrol system while reciprocating the velocity/force. Therefore, in order to solve the issue of active energy, the communication medium has been modelled as a Two-port network in which velocity/force move contrary to each other as depicted in Fig. 7. Each port corresponds to a real-time velocity/force signal 
and its conjugate delayed signal. The velocity force multiplication is power and the accumulation of this power over time results in energy. This modification of the communication medium is called the TDPN. TDPN acts in the same manner as the conventional communication medium but it assists in resolving the issue of random time delay inherent to a network. Instead of exchange of velocity/force, a non-conventional concept is used i.e. transfer of energy at each port. Positive and negative energies are segregated at each port. Positive energy is getting in while negative energy is getting out at each port. The analysis of energy is done with respect to input energy at the output of each port. PO calculates the net energy output at each port. Whenever PO finds out an active energy presence, then the relevant PC takes the corrective measures to dump extra active energy to make the system passive again. This leads to a stable telecontrol as shown in Fig. 8. This approach separates the energy flow in the backward and forward direction and there is no connection between these two quantities for passivity analysis of the whole system.

\section{A. Passivity of TDPN}

Figure 7 shows the velocities and forces entering and leaving a Two-port network. Power is the product of these two variables. The total power of the network can be written as given in (1).

$$
P_{N}(t)=P_{M}(t)+P_{S}(t)
$$

$P_{M}(t)(\mathrm{M}=$ master $)$ is the power on the master side of a Two-port network and $P_{S}(t)$ (S=slave) is the power on the slave side while $P_{N}(t)(\mathrm{N}=$ network $)$ is the power of the network. The energy at both sides of the network is described as

$$
\begin{aligned}
& E_{M}(t)=\int_{0}^{t} P_{M}(\tau) d \tau \\
& E_{S}(t)=\int_{0}^{t} P_{S}(\tau) d \tau \\
& E_{M}(t)=\int_{0}^{t} f_{M}^{\prime}(\tau) V_{M}(\tau) d \tau \\
& E_{S}(t)=\int_{0}^{t} f_{S}(\tau) V_{S}^{\prime}(\tau) d \tau
\end{aligned}
$$

$E_{M}(t), E_{S}(t)$ are the energies on master side and slave side respectively. Hence, to keep the network passive, the following condition should prevail.

$$
P_{N}(t) \geq 0, \quad \forall t \geq 0
$$

$E_{M}(t), E_{S}(t)$ are not available due to time delay simultaneously. Therefore, in order to solve this issue the positive and negative power has been taken at each port so that net energy can be calculated in the presence of delay. 
$P_{M}^{+}(t)=P_{M}(t) \quad \forall t \geq 0 \&$

$f_{M}^{\prime}(t) V_{M}(t) \geq 0$

$P_{M}^{-}(t)=-P_{M}(t) \quad \forall t \geq 0 \&$

$f_{M}^{\prime}(t) V_{M}(t) \leq 0$

$P_{S}^{+}(t)=P_{S}(t) \quad \forall t \geq 0 \&$

$f_{S}(t) V_{S}^{\prime}(t) \geq 0$

$P_{S}^{-}(t)=-P_{S}(t) \quad \forall t \geq 0 \&$

$f_{S}(t) V_{S}^{\prime}(t) \leq 0$

Both robots positive and negative energies are

$$
\begin{array}{ll}
E_{M}^{+}(t)=\int_{0}^{t} P_{M}^{+}(\tau) d \tau & \forall t \geq 0 \\
E_{M}^{-}(t)=\int_{0}^{t} P_{M}^{-}(\tau) d \tau & \forall t \geq 0 \\
E_{S}^{+}(t)=\int_{0}^{t} P_{S}^{+}(\tau) d \tau & \forall t \geq 0 \\
E_{S}^{-}(t)=\int_{0}^{t} P_{S}^{-}(\tau) d \tau & \forall t \geq 0
\end{array}
$$

$E^{\text {in }}(t)$ is positive and $E^{\text {out }}(t)$ is negative entering and leaving the port respectively at each side.

$$
\begin{array}{ll}
E_{M}^{\text {in }}(t)=E_{M}^{+}(t) & \forall t \geq 0 \\
E_{M}^{\text {out }}(t)=E_{M}^{-}(t) & \forall t \geq 0 \\
E_{S}^{\text {in }}(t)=E_{s}^{+}(t) & \forall t \geq 0 \\
E_{S}^{\text {out }}(t)=E_{M}^{-}(t) & \forall t \geq 0 \\
E_{N}(t)=E_{M}(t)+E_{S}(t)
\end{array}
$$

Overall net energy of whole system is given in (20).

$$
\begin{aligned}
& E_{N}(t)=E_{M}^{\text {in }}(t)-E_{M}^{\text {out }}(t)+ \\
& E_{S}^{\text {in }}(t)-E_{S}^{\text {out }}(t)
\end{aligned}
$$


$E_{M \rightarrow S}(t)$ is the energy from master haptic device to slave robot and $E_{S \rightarrow M}(t)$ from slave robot to haptic device.

$$
\begin{aligned}
& E_{N}(t)=E_{M \rightarrow S}(t)+E_{S \rightarrow M}(t) \\
& E_{M \rightarrow S}(t)=E_{M}^{\text {in }}(t)-E_{S}^{\text {out }}(t) \\
& E_{S \rightarrow M}(t)=E_{S}^{\text {in }}(t)-E_{M}^{\text {out }}(t)
\end{aligned}
$$

The network is passive until the (24) and (25) inequalities are satisfied.

$$
\begin{aligned}
& E_{M \rightarrow S}(t) \geq 0 \\
& E_{S \rightarrow M}(t) \geq 0
\end{aligned}
$$

Df is the delay time from master to slave called forward delay and $\mathrm{Db}$ is the backward delay from slave robot to haptic device. The net energies with a forward delay Df and a backward delay Db are given in (26) and (27).

$$
\begin{aligned}
& E_{M \rightarrow S}(t)=E_{M}^{\text {in }}\left(t-D_{f}\right)-E_{S}^{\text {out }}(t) \\
& E_{S \rightarrow M}(t)=E_{S}^{\text {in }}\left(t-D_{b}\right)-E_{M}^{\text {out }}(t)
\end{aligned}
$$

\section{B. Passivity observer}

$E_{M}^{O b s}(n), E_{S}^{O b s}(n)$ are observers at each side as given in (28) and (29). n represents the random time interval between two sample time. $\alpha_{M}$ in (28) is master controller and $\alpha_{S}$ in (29) is slave controller.

$$
\begin{aligned}
& E_{M}^{\text {obs }}(n)=E_{M}^{\text {obs }}(n-1)+\left(E_{M}^{\text {in }}\left(n-D_{f}\right)-\right. \\
& \left.E_{S}^{\text {out }}(n)+\alpha_{M}(n-1) V_{M}(n-1)^{2}\right) \\
& E_{S}^{O b s}(n)=E_{S}^{o b s}(n-1)+\left(E_{S}^{\text {in }}\left(n-D_{b}\right)-\right. \\
& \left.E_{M}^{\text {out }}(n)+\alpha_{S}(n-1) f_{S}(n-1)^{2}\right)
\end{aligned}
$$

\section{Passivity Controller}

To realize a stable telecontrol, it is necessary to dump active energy which is introduced by the communication medium into the telecontrol system. 


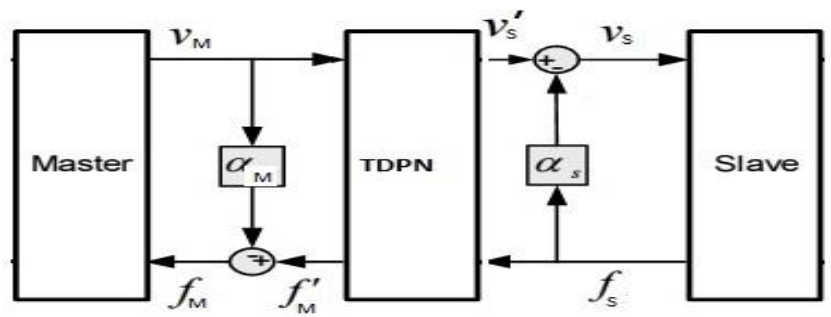

Figure 8. Master and slave controllers.

Designed $\alpha_{M}$ and $\alpha_{S}$ behave as dissipative elements on master and slave side of the TDPN respectively. The dissipation action reduces the effect of surplus energy to minimal. $\alpha_{M}, \alpha_{s}$ provide necessary controller action when the value of the observed energy is negative. The corrective measures are applied to velocity and force feedback as given in (32) and (33) and shown in Figure 8.

$$
\begin{aligned}
& \alpha_{M}(n)= \begin{cases}0 & \text { if } \quad E_{M}^{o b s}(n)>0 \\
-\frac{E_{M}^{O b s}(n)}{V_{M}^{2}(n)}\end{cases} \\
& \alpha_{S}(n)=\left\{\begin{array}{l}
0 \quad \text { if } \quad E_{S}^{O b s}(n)>0 \\
-\frac{E_{S}^{O b s}(n)}{f_{S}^{2}(n)}
\end{array}\right. \\
& f_{M}(n)=f_{M}^{\prime}(n)+\alpha_{M}(n) V_{M}(n) \\
& V_{S}(n)=V_{S}^{\prime}(n)+\alpha_{S}(n) f_{S}(n)
\end{aligned}
$$

\section{EXPERIMENTAL RESULTS}

The performance of passivity control with TDPN for telecontrol of mobile robot AutoMerlin has been plotted and presented in this section. The performance of the controller has been tested without and with the obstacles around the robot in the remote environment. The first test run was performed when the environment was free of obstacles. Fig. 9, shows offset between two velocities due to the time delay. The dots represent the random time delay between two sample times. It is vivid from Fig. 9, that there is no surge in slave velocity after passing through a communication channel with random delay. Blue plot is energy input and green plot energy output on master and slave side respectively in Fig. 10. The output energy has surge in it due to active energy. Hence, its value is greater than the input energy as depicted in the Fig. 10. The slave controller measures the active energy and diffuses its effect by dissipating it so that the forward communication always remains passive as drawn in Fig. 11, by the blue line. Force feedback is sent back from the remote environment to master haptic device. Fig. 12, is showing the force on the remote robot as a blue line and force feedback on the haptic device as a green 
line with the random delay indicated by dots. Fig. 13, shows the input energy entering the port from the slave side with the blue line and green line plot is the output energy on the other side. The surplus energy is being added by the network and due to this addition, the system output energy is greater than respective input energy. As the force on both sides is similar, the master controller is dissipating the effect of active energy as shown in Fig. 14. Whenever there is an active behavior by the network on either or both side the master and/or the slave controllers dissipate the same amount of active energy so that the system remains passive and hence stable.

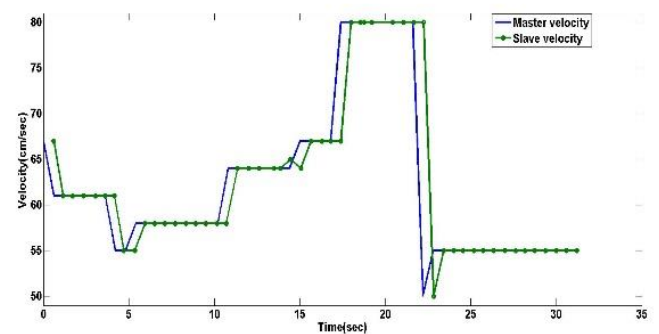

Figure 9. Linear velocities of both robots.

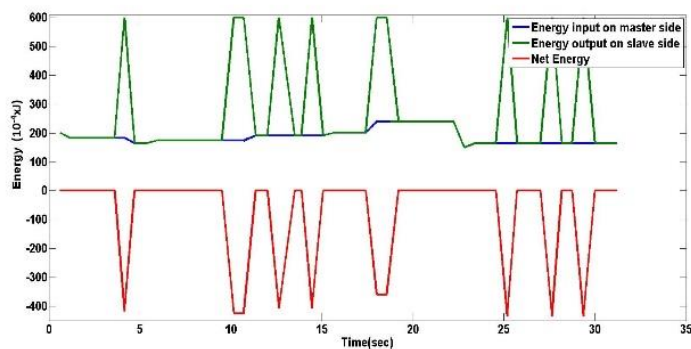

Figure 10. Energy flow master to slave.

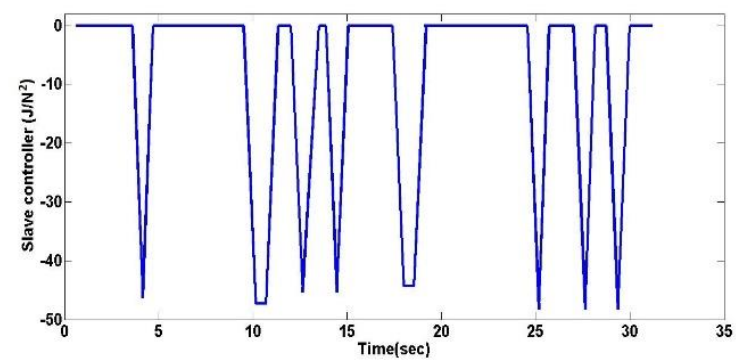

Figure 11. ${ }^{\alpha}$ controller

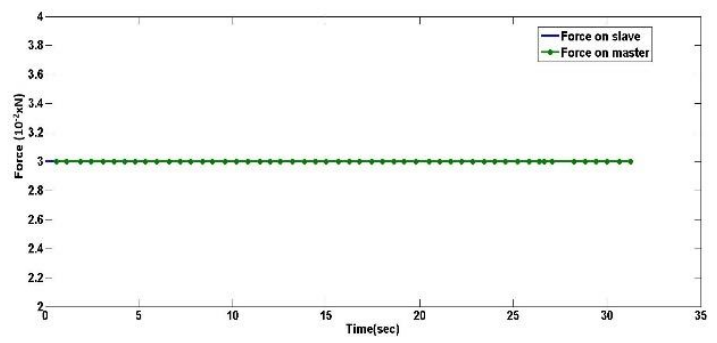

Figure 12. Force comparison on both robots. 


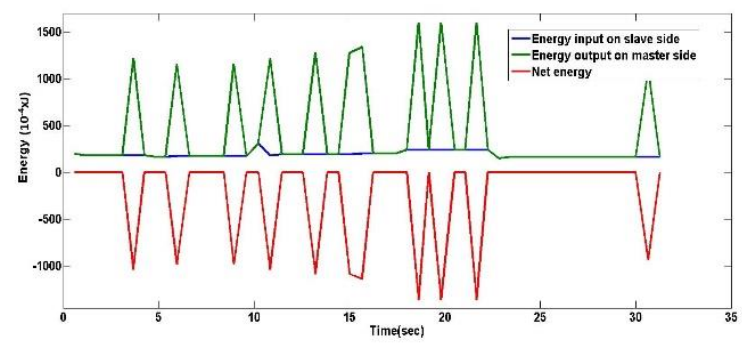

Figure 13. Energy flow slave to master.

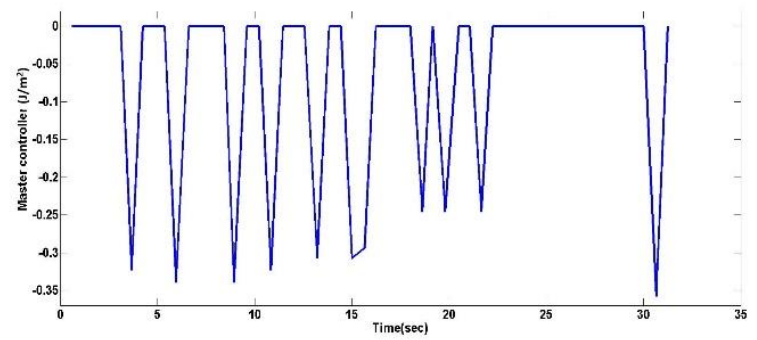

Figure 14. $\alpha_{M}$ controller.

The second test run was done when there were obstacles in the environment to evaluate the performance of the controller. The following plots show the performance of the controller with obstacles around the operational area of the remote slave robot. The Fig. 15, shows that there is no change in velocities and Fig .18, shows the force on both the slave robot and the master robot is the same. The dots indicate the random intervals. The rise in slave force is due to the unstructured environment with obstacles. This rise in force is vividly seen in Fig. 18. Similarly, the plots show that whenever there is an active behavior both $\alpha_{M}$ and $\alpha_{S}$ dissipate energy so that a stable and passive telecontrol can be performed

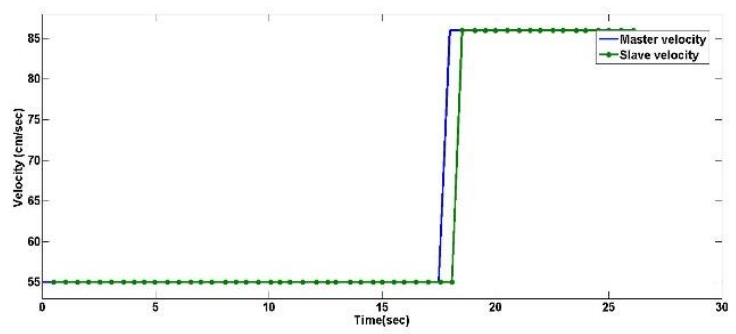

Figure 15. Linear velocities of both robots.

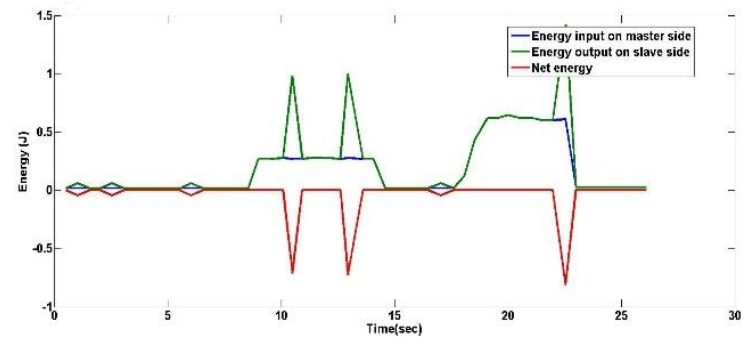

Figure 16. Energy flow master to slave. 


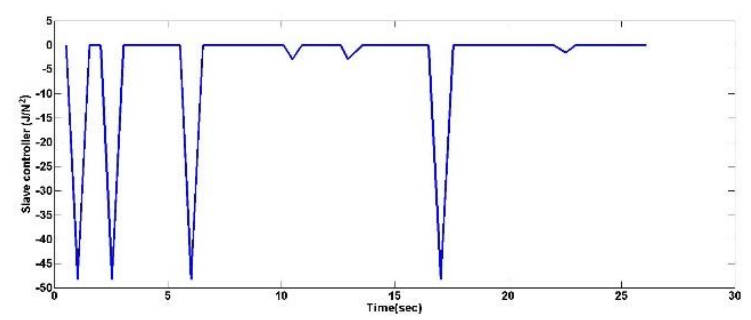

Figure 17. ${ }^{\alpha}$ controller.

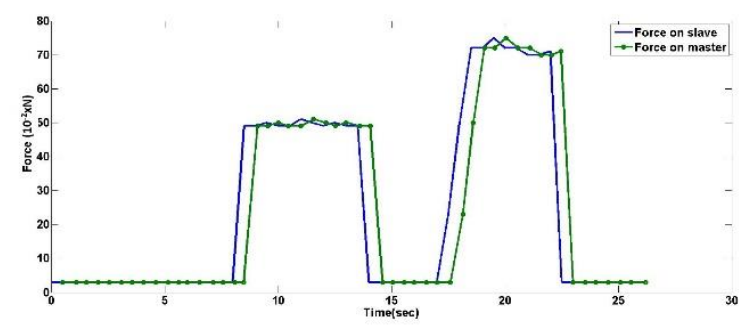

Figure 18. Force comparison on both robots.

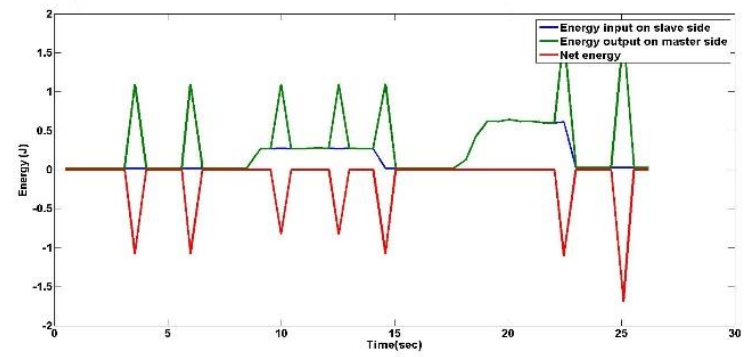

Figure 19. Energy flow slave to master.

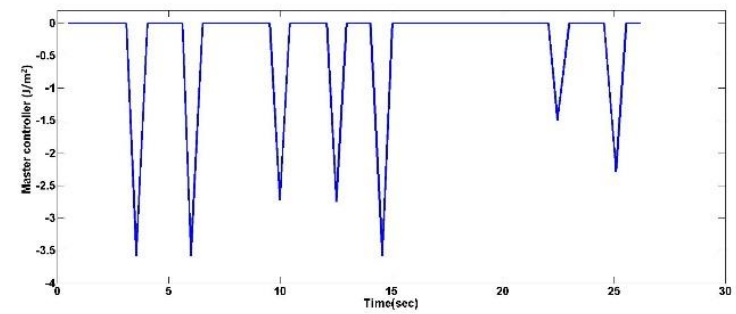

Figure 20. $\alpha_{M}$ controller.

\section{CONCLUSION AND FUTURE WORK}

The performance of the designed controller is excellent and it is keeping the system stable and passive all times. There is no compromise on unknown parameters because the controller is not based on the mathematical model of the system. It only functions when required. In order to extend this work, there are different tasks under planning like the addition of more robots for the telecontrol of a team of robots for multi-tasking.

\section{REFERENCES}

[1] A. Shahzad and H. Roth. "Teleoperation of mobile robot using event based controller and real time force feedback," Scientific Cooperations, International Workshops on Electrical and Computer Engineering Subfields, Koc University, Istanbul/Turkey, 22-23 August 2014. 
[2] A. Shahzad, R. Al-jarrah and H. Roth, "Telecontrol of AutoMerlin robot by employing fuzzy logic", International Journal of Mechanical Engineering and Robotics Research (IJMERR), Volume 5, No. 1, January, 2016.

[3] A. Shahzad, R. Al-jarrah and H. Roth, "Teleoperation of AutoMerlin by inculcating FIN algorithm", International Journal of Mechanical Engineering and Robotics Research (IJMERR), Volume 5, No. 1, January, 2016.

[4] A. Shahzad, H. Roth, "Bilateral telecontrol of AutoMerlin mobile robot", 9Th IEEE international conference on open source systems and technologies, Lahore, Pakistan, 17-19 December, 2015.

[5] V. Chawda and M. K. O’Malley," Position synchronization in bilateral teleoperation under time-varying communication delays," Ieee/asme transactions on Mechatronics, Volume 20, Issue 1, Feburary, 2015.

[6] R. Anderson and M. Spong, "Bilateral control of teleoperators with time delay,” IEEE Trans. Autom. Control, vol. 34, no. 5, pp. 494-501, May.1989.

[7] R. J. Anderson and M. W. Spong, "Asymptotic stability for force reflecting teleoperators with time delay,” Int. J. Robot. Res., vol. 11, no.2, pp.135-149, 1992.

[8] H. K. Khalil, Nonlinear Systems, vol. 3. Upper Saddle River, NJ, USA,Prenticehall, 2002.

[9] K. Hashtrudi-Zaad and S. E. Salcudean, "Analysis of control architectures for teleoperation systems with impedance/admittance master and slave manipulators," Int. J. Robot. Res., vol. 20, no. 6, pp. 419-445, 2001.

[10] A. Haddadi, "Stability, performance, and implementation issues in bilateral teleoperation control and haptic simulation systems," Ph.D. dissertation, Dept. Electr. Comput. Eng., Queen's Univ., Kingston, Canada, 2012.

[11] G. Niemeyer and J.-J. Slotine, "Stable adaptive teleoperation," IEEE J. Ocean. Eng., vol. 16, no. 1, pp. 152-162, Jan. 1991.

[12] R. Anderson and M. Spong, "Bilateral control of teleoperators with time delay," IEEE Trans. Autom. Control, vol. 34, no. 5, pp. 494-501, May.1989.

[13] P. Arcara and C. Melchiorri, "Control schemes for teleoperation with time delay: A comparative study,” Robot. Auton. Syst., vol. 38, no. 1, pp.49-64, 2002.

[14] P. F. Hokayem and M. Spong, "Bilateral teleoperation: An historical survey," Automatica, vol. 42, no. 12, pp. 2035-2057, 2006.

[15] E. Nuno, L. Basa nez, and R. Ortega, "Passivity-based control for bilateral teleoperation: A tutorial,” Automatica, vol. 47, no. 3, pp. 485-495, 2011.

[16] J. Ryu, D. Kwon, and B. Hannaford, "Stable teleoperation with time domain passivity control,” IEEE Trans. Robot. Autom., vol. 20, no.2, pp.365-373, Apr. 2004.J. Ryu, J. Artigas, and C. Preusche, "A passive bilateral control scheme for a teleoperator with time-varying communication delay," Mechatronics, vol. 20, no. 7, pp. 812-823, 2010.

[17] Y. Ye, Y.-J. Pan and T. Hilliard, "Bilateral teleoperation with time-varying delay: A communication channel passification approach," IEEE/ASME Trans. Mechatronics, vol. 18, no. 4, pp. 1431-1434, Aug. 2013. 
